\title{
Savoirs mobilisés par les élèves dans des cafés science: grille de caractérisation issue d'une étude internationale
}

\author{
Claire Polo \\ École normale supérieure de Lyon \\ claire.polo@ens-lyon.fr \\ Christian Plantin \\ École normale supérieure de Lyon \\ christian.plantin@univ-lyon2.fr \\ Kristine Lund \\ École normale supérieure de Lyon \\ kristine.lund@univ-lyon2.fr \\ Gerald Niccolai \\ École normale supérieure de Lyon \\ gerald.niccolai@ens-lyon.fr
}

\begin{abstract}
Co-développé par une association de médiation scientifique et un laboratoire de recherche, le dispositif pédagogique YouTalk se situe à la croisée de plusieurs préoccupations de la littérature récente en éducation scientifique. Ce café scientifique adapté pour le milieu scolaire permet aux élèves d'argumenter à propos d'une controverse socio-scientifique, ici la gestion de l'eau potable. À partir des vidéos de 10 cafés menés au Mexique, aux États-Unis, et en France, une caractérisation fine des éléments de savoir-croyance $(\mathrm{ESaC})$ utilisés par les élèves a été réalisée, sur cinq plans : leur niveau logique, leur niveau de généralité, la facilité avec laquelle ils sont comparables aux savoirs de références, leur position didactique vis-à-vis de ces derniers, et leur(s) source(s). La prédominance, au niveau logique, d'ESaC concernant une relation plutôt que la simple mention d'ESaC-objets témoigne de l'entrée des élèves dans un véritable processus de raisonnement. Une tendance à formuler des $\mathrm{ESaC}$ de plus haut degré de généralité lors de discussions en classe entière indique la complémentarité entre les activités se déroulant à ce niveau et le discours en petits groupes. Enfin, l'attention portée aux sources des ESaC répertoriés, et à leur relation aux savoirs de référence, nous informe sur l'intérêt d'un tel dispositif vis-à-vis des missions plus classiques de l'école.
\end{abstract}

Mots-clefs : savoir, argumentation, controverse, éducation à l'environnement

\section{Characterizing the Knowledge Used by Students in Scientific Cafés: Analytical Framework Based on an International Study}

The YouTalk educational activity, co-designed by a research laboratory and an association specialized in scientific mediation, was defined at the crossroads of several problems of the literature of science education research. This school scientific café allows the students to argue about a socio-scientific issue, here drinking water management. On the basis of 10 videotaped cafés organized in Mexico, the US, and France, the elements of knowledge-belief (EKoB) used by the students are described along five dimensions: logical level, degree of generality, comparability to knowledge of reference, didactical position in relation to this knowledge, source. In terms of logical level, most of the EKoB corresponds to relations, which indicates students' engagement into a reasoning process. The students also tend to use $\mathrm{EKoB}$ of a higher degree of generality during class discussion than the ones they use in small groups. This result is consistent with the initial hypothetized complementarity between class-level and group-level discussions that inspired the pedagogical design. Last but not least, attention paid to the source(s) of the identified EKoB and 
their relation to knowledge of reference is key to inform us about the interest of such activity for traditional missions of formal education.

Keywords: knowledge, argumentation, controversy, environmental education

\section{Introduction}

La littérature internationale en didactique des sciences est marquée d'une part par un intérêt croissant pour la place de l'argumentation en classe de science (par exemple Andriessen, Baker \& Suthers, 2003 ; Boulter \& Gilbert, 1995 ; Buty \& Plantin, 2008 ; De Vries, Lund \& Baker, 2002 ; Driver, Newton \& Osborne, 2000 ; Erduran \& Jiménez-Aleixandre, 2007 ; Newton, Driver \& Osborne, 1999), et, d'autre part, par l'émergence de nouveaux objets d'enseignement, nommés pas la didactique des sciences anglo-saxonne socio-scientific issues (par exemple Kolsto, 2001; Zeidler et al., 2005). Ces deux évolutions sont associées à l'approche par compétences, et s'inscrivent dans une vision renouvelée de l'enseignement des sciences, où l'important n'est plus seulement d'apprendre des savoirs mais aussi de se former aux méthodes de production du savoir, et d'acquérir des capacités de réflexion critique et de contextualisation sociale de ces savoirs.

Pensées légèrement différemment en France (Simonneaux \& Simonneaux, 2009), notamment sous la dénomination de «controverses socio-scientifiques»(Albe, 2009b, p. 28), ces questions sont complexes, n'admettent pas une réponse unique, ni ne se laissent appréhender par le seul point de vue technoscientifique. De plus, se construire une opinion à leur propos suppose de mobiliser des savoirs variés, ainsi que de réaliser des choix politiques et éthiques, faisant intervenir valeurs, intérêts, et émotions. Un consensus existe sur l'importance de former les citoyens à de telles questions, de plus en plus prégnantes dans notre société. Ici, éducation scientifique et éducation à la citoyenneté se rejoignent.

Cependant, la scolarisation de tels objets pose des difficultés (Albe, 2009a), en termes de formation des enseignants, et de craintes liées à l'appréhension de sujets politiquement " chauds ». Certains enseignants sont sceptiques quant à l'intérêt de débats socio-scientifiques, perçus comme susceptibles de conduire à des batailles d'opinions stériles. Il y a donc un enjeu fort à développer des outils pour caractériser et évaluer les contenus didactiques construits dans de tels dispositifs.

Tant pour son contenu que pour les pratiques pédagogiques qui y sont associées (analyse de médias, débats, jeux de rôle), le traitement de controverses socio-scientifiques constitue un champ privilégié pour l'établissement de partenariats entre l'école et d'autres acteurs éducatifs, notamment dans le champ de la culture scientifique et technique. Historiquement, les cafés des sciences ont constitué un mode emblématique de discussion de ces questions, pour un public adulte. Dès 2007, le laboratoire ICAR (Interactions Corpus Apprentissages Représentations) s'est inspiré de ce modèle pour concevoir des situations didactiques à destination d'un public scolaire (Niccolai, Badreddine \& Buty, 2010). Parallèlement, l'Association Rhône-Alpes des Petits Débrouillards (ARAPD) a été amenée à développer ses interventions scolaires sur des questions environnementales, ainsi qu'à concevoir de nouveaux outils pour appréhender les relations science-société (Landais, 2011). A partir de 2011, un partenariat entre ces deux institutions a permis l'élaboration d'un nouveau dispositif de "café scientifique junior », le YouTalk. Il consiste en une intervention dans la classe, ponctuelle, durant environ 2 heures. Une première thématique, la gestion de l'eau potable, a été abordée sur ce format d'éducation « semi-formelle » dans plusieurs pays, entre 2011 et 2014.

À partir des vidéos de 10 cafés menés au Mexique, aux États-Unis, et en France, une étude approfondie des argumentations développées par les élèves a été réalisée, avec une description fine des ressources argumentatives utilisées, distinguant normes, émotions, et savoirs ${ }^{1}$ (Polo, 2014). Cet article vise à présenter les principaux outils développés à cette occasion pour caractériser les savoirs émergeant dans cette situation, outils éprouvés sur différents terrains. En effet, la conception didactique traditionnelle du savoir scolaire ne permet pas d'appréhender les éléments hybrides mobilisés par les élèves dans une telle situation. Comment saisir les spécificités des contenus construits dans ces cafés? Nous proposons de nouveaux outils analytiques pour ce faire, et pour problématiser leur lien avec les savoirs de référence. Au-delà de ce dispositif particulier, il s'agit de proposer un cadre analytique utile pour la description de toute situation éducative située dans un " entre-deux » associant dimension médiatique et dimension didactique. Ici, le partenariat s'inscrit dans une volonté de médiatisation de l'espace scolaire, dans un espace-temps limité, permettant d'enrichir le traitement d'une controverse socio-scientifique. Sa didactisation se trouve facilitée par l'aspect informel du dispositif, permettant une meilleure articulation entre les savoirs et savoir-faire en jeu et la vie quotidienne et l'implication sociale des

$1 \quad$ Le terme de normes englobe ici aussi bien les valeurs, ou normes fondamentales, que les normes procédurales (ayant trait au contrat de communication ainsi qu'aux règles argumentatives employées dans la situation). Les émotions sont plutôt appréhendées, en tant que ressources argumentatives, pour leur rôle dans la schématisation des objets de discours, et l'orientation argumentative globale du propos, ou emotional framing. Quant à la ressource savoir, sur laquelle porte cet article, elle est plus précisément définie et problématisée en 2.2 et 2.3 . 
élèves, et, par conséquent, une meilleure compréhension de la complexité de la question. À ce titre, nous estimons également que ce cadre analytique peut être utile pour appréhender le contenu de toute discussion à visée didactique portant sur des controverses socio-scientifiques.

Dans une première section, nous précisons le contexte de ce travail et la situation étudiée (1). Au fil de la section suivante (2), nous présentons notre angle problématique et les catégories d'analyse développées, en lien avec la littérature préexistante. Enfin, nous illustrons le fonctionnement de la grille proposée à partir de données issues des trois pays (3). En conclusion, nous mettons en perspective ces résultats avec les missions attribuées à l'école et à ses partenaires scientifiques.

\section{YouTalk: projet, dispositif pédagogique, situations didactiques}

Cette première section détaille le contexte de l'étude et précise la nature du corpus d'analyse. Une première sous-partie aborde le travail partenarial de conception du dispositif pédagogique (1.1.), qui est ensuite présenté précisément (1.2.). Enfin, une dernière sous-partie insiste sur la diversité des situations didactiques réelles auxquelles nous avons eu affaire (1.3.).

\subsection{Conception à l'interface recherche - éducation non-formelle - écoles}

Un partenariat entre le laboratoire ICAR et l'ARAPD a été scellé en 2011, financé par le dispositif régional "Université solidaire et citoyenne ». Le cahier des charges établi pour la conception d'un nouveau café scientifique junior conjuguait objectifs relevant de l'objet social de l'association, objectifs propres aux établissements scolaires, et contraintes liées à la constitution d'un corpus de recherche. Une phase de réunions préliminaires a permis de clarifier les référentiels de chacun des acteurs institutionnels, et a conduit à la détermination d'objectifs pédagogiques communs (cf. annexe 1). Ce processus collaboratif s'est appuyé sur des personnalités jouant le rôle de «passeurs » (brokers) entre les différents univers professionnels concernés (Polo, $2013)$ : chercheurs également membres de l'ARAPD, responsable du projet côté associatif titulaire d'un master de didactique, ingénieur-chercheur pour la captation audiovisuelle, chercheure-formatrice à l'animation ${ }^{2}$, enseignants familiers de la coopération avec des institutions de recherche. En effet, leur participation a favorisé l'introduction de pratiques de médiation scientifique dans le partenariat recherche-écoles, et inversement, permis une relative formalisation des pratiques médiatiques pour leur adaptation à différents milieux scolaires (Wenger, 2000, p. 235). Ils ont joué le rôle typique de créateurs d'un langage commun (Meyer, 2010, p. 120-121), notamment concrétisé dans la seconde phase de travail, correspondant à la conception du dispositif pédagogique. Ce travail commun a été le lieu de façonnage d'objets intermédiaires (Vinck, 2009), et le diaporama servant de support à l'animation a servi d'incarnation à certains objets-frontières (Star \& Griesemer, 1989).

De plus, dès son origine, le YouTalk a été pensé pour être adapté à l'international. Très vite, d'autres partenaires ont donc pris part au projet. Ainsi, la collaboration avec l'association de médiation scientifique mexicaine Pandillas Científicas a influencé le choix de la thématique développée en priorité, la gestion de l'eau potable. En outre, ce thème présentait l'intérêt de recouvrir à la fois des enjeux globaux et des problèmes concernant le quotidien des élèves. Des enseignants de différentes disciplines ont été associés au projet, en amont, et ont participé à l'adaptation du dispositif au contexte local, pour le Mexique et les États-Unis.

Notre démarche de conception appréhendait le dispositif pédagogique à construire dans une double dimension : d'une part l'aboutissement à une séquence d'activités, et, d'autre part, la mise en place de principes spécifiques d'organisation des interactions entre les participants. Nous avons donc conçu un macro-script, c'est-à-dire un ensemble d'instructions qui définissent les différentes phases, rôles, et séquences d'activités autour d'un outil pédagogique (Weinberger et al., 2005 ; Tsovaltzi et al., 2008). Cette perspective rejoint l'approche de l'écologie de l'apprentissage (Cobb et al., 2003 ; Albe, 2009b).

Un élément-clef de ce macro-script concerne le fait que l'ensemble du café est animé par des élèves plus âgés (équivalent du lycée) pour des élèves de fin de collège. L'animation est assurée en binôme, avec une personne chargée de la plupart des échanges avec la classe, et une personne ayant davantage un rôle d'observation. La dynamique partenariale d'hybridation des pratiques éducatives et de recherche imprègne ainsi le dispositif luimême, notamment dans la façon de percevoir les élèves, dépositaires de différents statuts. Ainsi, les participants au café sont considérés comme des élèves, notamment par les enseignants, mais ils sont plutôt sollicités dans le dispositif en tant que citoyens en herbe, et considérés par les animateurs comme des pairs. Les animateurs, lycéens, se retrouvent à jouer d'une certaine façon un rôle de «passeurs » également, entre le vécu commun du

2 Cette personne, en temps que formatrice, a assuré la formation des lycéens à l'animation du dispositif, observé les cafés en leur procurant parfois des conseils en temps réel, en aparté, et a réalisé avec l'équipe de lycéens-animateurs des bilans intermédiaires et finaux (après chaque café, et en fin d'intervention). Ce travail de coaching a été documenté par des enregistrements audio et vidéo, mais il ne fait pas l'objet de cet article. 
métier d'élève qu'ils partageaient avec les collégiens, et le travail d'animation pour lequel ils ont reçu une formation spécifique d'une journée.

\subsection{Le café scientifique YouTalk}

Le YouTalk se définit comme un dispositif d'éducation semi-formel, organisé à l'école, mais hors discipline spécifique, et hors système d'évaluation. Il s'agit d'une intervention ponctuelle de 90 minutes aux États-Unis, 110 minutes en France et au Mexique. Les élèves sont disposés par groupes de 3 à 5 autour d'une table, afin de favoriser les échanges en petits groupes.

La séquence pédagogique comprend 5 phases : introduction, trois phases thématiques, conclusion (récapitulées en annexe 2). Le café est rythmé par la projection d'un diaporama affichant un questionnaire à choix multiple ${ }^{4}$.

Une brève introduction permet d'annoncer la thématique et d'expliciter les règles du jeu. Une attention particulière est portée à la distinction entre les questions d'opinion (QO) qui n'ont pas de bonne réponse, et sont débattues, et les questions de connaissance (QC), pour lesquelles une bonne réponse est identifiée ${ }^{5}$. La question principale (QP) est présentée, et l'opinion initiale de chaque élève est recueillie anonymement.

Cette QP est ensuite explorée via trois phases thématiques comprenant chacune plusieurs QC puis une QO finale. Les QC donnent lieu à de brefs échanges en groupe, puis à un sondage individuel anonyme, via un dispositif électronique ou papier. La bonne réponse est donnée, puis justifiée. Les QO, dans une autre couleur, sont traitées autrement : les élèves ont davantage de temps pour en discuter et doivent se mettre d'accord dans leur groupe. Ce positionnement collectif est exprimé par l'affichage d'une lettre en carton sur un pupitre. Ensuite a lieu un débat en classe entière. Pendant qu'un animateur facilite la discussion, l'autre prend des notes au tableau. Enfin, les élèves se positionnent individuellement et anonymement sur la QO (vote papier ou électronique).

En conclusion, l'animateur-observateur fait une rapide synthèse des débats, puis la QP initiale est reposée, et traitée comme une QO, avec un temps de débat plus long.

\subsection{Situations pédagogiques}

De nombreux autres éléments caractérisent les situations pédagogiques étudiées dans ce corpus. Au total, les analyses présentées ici ont été réalisées à partir des enregistrements audiovisuels de 10 cafés, de 4 écoles : une école publique rurale mexicaine ( 2 cafés), une école privée, urbaine, mexicaine (2), un collège de centre-ville français (3), et une école américaine publique (3). Ces environnements diffèrent amplement aux plans géographique, linguistique, culturel, socio-économique, institutionnel. Ils présentent une diversité de pratiques éducatives routinières et de situations concernant la thématique abordée.

Ainsi, l'adaptation du dispositif à ces terrains ne correspond pas à une démarche expérimentale contrôlée où il serait possible de reproduire à l'identique une situation, en ne faisant varier que quelques facteurs. L'approche méthodologique consiste plutôt à décrire les pratiques observées en contextes authentiques. Dans cet article, la mobilisation de ce corpus international n'a pas de visée comparative forte, il s'agit simplement d'éprouver la robustesse de la grille analytique proposée en montrant son adéquation descriptive et explicative sur différents terrains. La section 2 précise cette démarche.

\section{Démarche analytique : appréhender des savoirs hétérogènes}

Dans cette partie méthodologique, nous présentons d'abord l'angle problématique et le corpus d'analyse constitué à cet effet (2.1.), puis les principes directeurs qui ont inspiré notre démarche, en lien avec la littérature correspondante (2.2.). Nous présentons enfin la grille analytique développée (2.3.).

\subsection{Problématique et corpus d'analyse}

À l'appui de cet important corpus, notre problème est d'identifier des dimensions structurantes permettant de caractériser les savoirs développés dans les discours des élèves. Tant les objets abordés que le dispositif

3 Nous faisons ici l'économie d'une présentation détaillée des motivations des choix d'ingénierie pédagogique, qui a été réalisée ailleurs (Polo, 2014, p. 88-100). Le diaporama utilisé dans chacun des pays comme support du café est également publié en ligne dans ce document (annexes, p. 14-25), et accessible librement ici : $<$ http://theses.univ-lyon2.fr/documents/lyon2/2014/polo c $>$.

$4 \quad$ Trop long pour être intégré ici, ce questionnaire est à la disposition du lecteur sur simple demande aux auteurs.

$5 \quad$ Présentées comme des devinettes, les QC portent sur des éléments de savoir stabilisés concernant le problème étudié, à savoir la gestion de l'eau potable. Cependant, plutôt que de présenter la réponse comme définitive et absolue, celle-ci est toujours expliquée en référence à une source précisément rapportée. 
pédagogique lui-même ont vocation à susciter chez eux des références à des savoirs et des croyances de nature et d'origine variées, tels qu'ils entrent en jeu dans la construction d'une opinion au sujet d'une controverse socioscientifique. Comment mettre de l'ordre dans le foisonnement d'éléments de savoir très hétérogènes présents dans chacun des cafés ? Quelles distinctions conceptuelles peuvent nous permettre de mieux les caractériser, à la fois dans une perspective d'identification des stratégies de raisonnement des élèves, et de comparaison aux savoirs de référence?

Afin d'appréhender le maximum d'éléments de savoirs, nous avons procédé à des enregistrements audiovisuels de ces cafés à plusieurs niveaux, aussi bien dans les petits groupes qu'en classe entière. En effet, les éléments mobilisés dans chacun de ces formats de participation sont susceptibles de varier. Nous disposons pour chaque café de 4 à 6 vues ( 2 à 4 vues focalisées sur des groupes, une vue de l'ensemble de la classe, et un enregistrement d'écran correspondant à ce qui est projeté). Les analyses réalisées portent donc à la fois sur les échanges en classe entière et en petit groupe. De plus, chacune de ces vues a été balisée, à l'aide du logiciel Transana, afin de pouvoir facilement accéder à une étape précise de la séquence pédagogique. Étant donné notre intérêt pour l'argumentation à propos de questions socio-scientifiques, cela nous a permis de focaliser l'analyse sur les discours produits pour la QP, et, pour 6 des 10 cafés (1 de chacune des écoles mexicaines, 2 pour les écoles française et américaine), pour les trois QO. Pour ces extraits, nous disposons d'une transcription verbale intégrale des échanges, suivant les conventions définies ICOR ${ }^{6}$.

\subsection{Langage descriptif : positionnement théorique et démarche empirique}

Nous présentons ici les principes qui ont guidé notre analyse : ne pas chercher à séparer, sur des critères externes, ce qui relève du savoir ou de la croyance, dans le discours des élèves (2.2.1.), mais plutôt identifier empiriquement des éléments de classification de ces savoirs-croyances (2.2.2.).

\subsubsection{Un continuum savoirs-croyances}

Scolariser les questions socio-scientifiques, c'est inviter les connaissances profanes à l'école, c'est-à-dire, étymologiquement, celles qui seraient « hors du temple », hors systèmes d'institutionnalisation et de légitimation classique du savoir (Albe, 2009a, p. 129). Ainsi, il serait inadéquat de chercher à caractériser ces éléments hybrides, en seule référence à la catégorie traditionnelle de "savoirs », définie plus étroitement. En réalité, les objets qui nous intéressent ici sont plutôt des « blocs de savoir-croyance ».

En effet, les éléments que nous cherchons à répertorier et caractériser ne correspondent pas à des « savoirs savants », au sens de savoirs institutionnalisés et validés par la communauté scientifique. Il ne s'agit pas non plus de « savoirs scolaires », issus d'une transposition didactique (Chevallard, 1992). Si les élèves, dans ces cafés scientifiques juniors, s'appuient bien sur des informations qu'ils présentent comme des vérités factuelles, celles-ci appartiennent plutôt à ce que Beitone et Legardez (1995) appellent le «système de représentations-connaissances» des acteurs, décrit par Simonneaux en ces termes : "il s'agit d'un agrégat comprenant des opinions, des croyances, des attitudes, des informations issues de diverses sources (dont la vulgarisation scientifique), des résidus d'apprentissages scolaires antérieurs, des représentations sociales » (2006, p. 46). Nous nous intéressons donc ici à ces éléments de savoir-croyance (désormais ESaC).

De plus, l'analyse d'interactions argumentatives authentiques ne permet pas d'isoler ce qui relève de la croyance et du savoir. En discours, il s'agit dans les deux cas d'éléments non contestés, présentés comme des évidences partagées. Nous faisons donc l'hypothèse d'un continuum entre savoirs et croyances.

\subsubsection{Une démarche empirique inspirée de la grounded theory}

Plutôt que de chercher à "faire rentrer » les énoncés repérés, artificiellement, dans des catégories théoriques préexistantes, nous avons cherché, empiriquement à identifier des éléments récurrents, selon la démarche de la grounded theory (Charmaz, 2006). Tout d'abord, les ESaC ont été regroupés par contenu, permettant d'identifier la récurrence d'informations similaires, et d'en dresser un inventaire. Chaque $\mathrm{ESaC}$ apparaît dans le corpus sous la forme d'une ou plusieurs occurrence(s) précise(s), des énoncé(s). Chaque énoncé peut renvoyer à un ou plusieurs ESaC. On peut ainsi regrouper et compter les énoncés renvoyant au même ESaC.

Cette première confrontation aux données a conduit à constater une très grande hétérogénéité parmi les $\mathrm{ESaC}$ répertoriés, à différents niveaux : taille (durée, matériel verbal), registre de langue, degré d'explicite (vocabulaire spécialisé ou paraphrase), etc. Nous avons ainsi défini cinq catégories analytiques, décrites dans la section 2.3.

\footnotetext{
${ }^{6}$ Elles sont entièrement détaillées dans la section « conventions de transcription » en ligne : < http://icar.univlyon2.fr/projets/corinte/>.
} 


\subsection{Grille d'analyse : 5 dimensions des éléments de savoir-croyance (ESaC)}

Nous avons développé une classification des ESaC selon cinq dimensions : niveau logique, niveau de généralité, comparabilité aux savoirs de référence, distance vis-à-vis de ces savoirs, source.

\subsubsection{Niveau logique}

Une première composante, permettant de mettre de classer ces éléments très hétérogènes, a trait au niveau logique considéré. Dans la logique classique, l'argumentation est définie par trois «opérations de l'esprit », agissant à des niveaux différents : l'appréhension porte sur la définition des êtres et les objets, le jugement affirme quelque chose à leur propos, aboutissant à une proposition; et le raisonnement lie les propositions entre elles. Ces opérations correspondent, au plan langagier, à la référence, à la prédication et à l'argumentation (Plantin, 2016, p. 282). Pour expliquer la construction des schématisations, intrinsèquement argumentatives, Grize les décompose également en opérations successives : 1) opération de constitution des notions primitives en objets de discours ou classes-objets ; 2) opération de détermination, aboutissant à des prédications ; 3) opération d'énonciation (prise en charge de la prédication par un sujet), aboutissant aux énoncés; 4) opérations de configuration établissant des relations entre les énoncés (Plantin, ibid., p. 424 ; Grize, 1990, p. 65-77). Les ESaC repérés dans notre corpus relèvent de «niveaux logiques » ou opérations cognitivo-langagières diverses. Ainsi, on peut distinguer les ESaC-objets des ESaC-relations. Les ESaC-objets portent sur la définition des « êtres » et des «objets », ne se limitant pas à la référence, mais pouvant inclure également des propositions issues de l'opération de prédication, et même envisager des relations potentielles avec d'autres êtres ou objets lorsqu'ils correspondent à des topoï constitutifs de l'identité de l'«objet» ou de l'«être » en question. Un ESaC-objet porte sur un objet et/ou ce que Grize nomme son «faisceau »: "J'appelle faisceau d'objet un ensemble d'aspects normalement attachés à l'objet. Ses éléments sont de 3 espèces : des propriétés, des relations, et des schèmes d'action. » (Grize, 1990, p. 78). Les ESaC-relations, eux, se situent à un autre niveau, établissant des liens entre ces êtres (faisceau inclus) et/ou ces objets (faisceau inclus). Les jugements portant sur ces objets (faisceau inclus) et/ou ces êtres (faisceau inclus) sont également des ESaC-relations, au sens où ils établissent implicitement un lien entre ces êtres ou objets et l'énonciateur.

\subsubsection{Niveau de généralité}

Les $\mathrm{ESaC}$ répertoriés se distinguent également sur le plan de leur niveau de généralité. Certains portent sur des points très précis, par exemple le prix d'une bouteille d'eau à l'épicerie du coin, tandis que d'autres concernent de grandes généralités, comme l'évolution mondiale du prix de l'eau.

\subsubsection{Comparabilité aux savoirs de référence}

De même, ces ESaC diffèrent par leur caractéristique d'être plus ou moins traduisibles dans le langage des savoirs de référence, et donc plus ou moins comparables aux résultats connus de la science ou à leur version vulgarisée. Certains $\mathrm{ESaC}$ ne se laissent pas aisément juger à l'aune de résultats scientifiques : leur nature floue ne permet pas de dire s'ils sont compatibles avec ces savoirs, sans interprétation excessive. Ces éléments sont qualifiés de doxaux, en référence à la notion de doxa.

\subsubsection{Position didactique vis-à-vis des savoirs de référence}

Quatrième axe structurant, lorsqu'ils se prêtent à une traduction ou une approximation dans le langage des savoirs de référence, les ESaC peuvent constituer plutôt un terreau favorable ou plutôt un obstacle à l'apprentissage de ces savoirs. Bien entendu, cette dichotomie est relative, et dépend des objectifs d'apprentissages et des contextes didactiques. Il ne s'agit pas de définir un élément de savoir comme étant par nature vrai ou faux, mais comme plus ou moins facilitateur de l'apprentissage des savoirs de référence correspondants, lorsque ceux-ci sont identifiables. Lorsqu'un ESaC supporte aisément la comparaison avec des savoirs de référence, et va dans le même sens, il est qualifié de quasi savant. A l'inverse, certains ESaC sont susceptibles de faire obstacle à l'apprentissage de ces savoirs. Lorsqu'ils constituent, en eux-mêmes, des contrevérités évidentes, ils sont qualifiés d'erronés. Cependant, la plupart des ESaC qui ne sont pas quasi savants ne sont pas pour autant erronés. Nombre d'entre eux, indépendamment de leur degré de véracité, peuvent constituer des obstacles à l'apprentissage de savoirs de référence, car ils véhiculent ou renforcent des représentations simplistes rendant difficile l'acquisition d'une représentation plus complexe et savante de l'objet concerné. Ils sont alors qualifiés de trompeurs. 


\subsubsection{Sources}

Enfin, les ESaC peuvent provenir d'une multiplicité de sources. Sa source constitue un des rares indices du positionnement d'une information sur l'axe savoir-croyance. Cependant, celle-ci est rarement explicitée. Dans notre corpus, six sources ont été identifiées :

1) l'expérience directe, conformément au constat d'Albe : «l'expérience personnelle apparaît comme un moyen pour interpréter et problématiser la controverse, une référence pour argumenter et justifier une position » (2006, p. 105);

2) le témoignage d'autrui ;

3) des « résidus d'apprentissages scolaires antérieurs » (Simonneaux, 2006, p. 46);

4) les « résidus » de connaissances abordées lors des QC antérieures du café ;

5) l'autorité parentale ;

6) les médias.

Les niveaux logiques et niveaux de généralités nous informent des formes de raisonnement des élèves, et notamment de leur passage à une forme d'abstraction et de généralisation. Les trois autres dimensions concernent plutôt le lien entre ces $\mathrm{ESaC}$ et les savoirs de référence, et permettent de problématiser le passage des uns aux autres. Le tableau 1 récapitule ces différentes dimensions en incluant quelques exemples.

\begin{tabular}{|c|c|c|c|c|}
\hline \multicolumn{5}{|c|}{ Caractérisation des éléments de savoir-croyance $(\mathrm{ESaC})$} \\
\hline \multirow[t]{2}{*}{ Source(s) } & \multirow[t]{2}{*}{ Niveau logique } & \multirow{2}{*}{$\begin{array}{l}\text { Degré de } \\
\text { généralité }\end{array}$} & \multicolumn{2}{|c|}{ Relation aux savoirs de référence } \\
\hline & & & Comparabilité & $\begin{array}{l}\text { Position } \\
\text { didactique }\end{array}$ \\
\hline \multirow{4}{*}{$\begin{array}{l}\text { 1. Expérience directe } \\
\text { 2. Témoignage } \\
\text { 3. Résidus de savoirs } \\
\text { scolaires } \\
\text { 4. Résidus des QC } \\
\text { antérieures du café } \\
\text { 5. Discours } \\
\text { parentaux } \\
\text { 6. Discours } \\
\text { médiatiques }\end{array}$} & \multirow{3}{*}{$\begin{array}{l}\text { ESaC-objets } \\
\text { Définition des } \\
\text { êtres et objets et } \\
\text { leur } \\
\text { «faisceau » } \\
\text { (Grize, 1990) } \\
\text { La Terre est } \\
\text { surtout } \\
\text { recouverte de } \\
\text { mers }\end{array}$} & \multirow[t]{3}{*}{$\begin{array}{l}\text { Précis } \\
\text { Prix d'un } \\
\text { jerricane d'eau } \\
\text { à l'épicerie du } \\
\text { coin }\end{array}$} & \multirow[t]{3}{*}{$\begin{array}{l}\text { ESaC non } \\
\text { doxaux } \\
\text { Il y a un trou } \\
\text { dans la couche } \\
\text { d'ozone }\end{array}$} & $\begin{array}{l}\text { ESaC quasi } \\
\text { savants } \\
\text { Plus l'eau est } \\
\text { rare, plus elle } \\
\text { est chère }\end{array}$ \\
\hline & & & & $\begin{array}{l}\text { ESaC } \\
\text { trompeurs } \\
\text { L'eau va } \\
\text { devenir la } \\
\text { nouvelle } \\
\text { monnaie }\end{array}$ \\
\hline & & & & $\begin{array}{l}\text { ESaC erronés } \\
\text { Dubaï est en } \\
\text { Inde }\end{array}$ \\
\hline & $\begin{array}{l}\text { ESaC- } \\
\text { relations (ou } \\
\text { lois) } \\
\text { Relations entre } \\
\text { êtres et objets } \\
\text { On a besoin } \\
\text { d'eau pour } \\
\text { vivre }\end{array}$ & $\begin{array}{l}\text { Généraux } \\
\text { Ce qui est vrai } \\
\text { aujourd'hui } \\
\text { sera vrai } \\
\text { demain }\end{array}$ & $\begin{array}{l}\text { ESaC doxaux } \\
\text { Les gens sont } \\
\text { égoïstes }\end{array}$ & \\
\hline
\end{tabular}

Tableau 1 : caractérisation des éléments de savoir-croyance : 5 dimensions exemplifiées

\section{Fonctionnement de la grille analytique sur trois terrains}

Dans cette partie, nous présentons d'abord les ESaC les plus fréquents, pour l'ensemble du corpus, et les énoncés associés, afin de donner à voir comment ils sont repérables dans des discours authentiques (3.1.). Les résultats de l'inventaire réalisé par corpus national sont ensuite mis en perspective à l'aide de la grille d'analyse (3.2.).

3.1. Des énoncés authentiques à un inventaire des $\mathrm{ESaC}$

L'annexe 3 présente tous les $\mathrm{ESaC}$ ayant au moins 3 occurrences pour au moins une des quatre écoles étudiées. Dans cette section, nous nous focalisons sur quelques-uns de ces éléments, les trois plus fréquents pour chaque corpus national. Afin de donner à voir les énoncés authentiques à partir desquels un même ESaC est identifié, nous en reproduisons un pour chaque école dans laquelle il apparaît. Cet accès au discours des élèves lui-même illustre le fonctionnement d'une telle analyse, et la nature très hétérogène des données verbales travaillées. 
Dans le corpus mexicain, l'ESaC le plus fréquent est les riches auront plus accès à l'eau. Cinq occurrences correspondent à l'école publique, avec par exemple :

MON sólo los ricos van a tener

(seuls les riches vont [en] avoir)

Pour l'école privée, 19 énoncés s’y réfèrent, comme :

MIG sólo la gente rica va a tener más disponibilidad al agua

(seuls les gens riches vont avoir plus accès à l'eaul)

Cet $\mathrm{ESaC}$ est également le plus fréquent dans le corpus américain, avec 21 occurrences, dont :

BRE the riches get the water and: no matter where you are on the globe if it's a poor area unfortunately

(les riches obtiennent l'eau $\backslash$ et: peu importe où tu es sur le globe si c'est un endroit pauvre

malheureusement)

On retrouve encore dans les données françaises (6 occurrences) :

PHI les pauvres n'auront pas de auront pas d'eau potable 'fin ou de l'eau de la mauvaise qualité et ceux qui [...] sont riches eb ben ils auront d'la bonne qualitê

Le second $\mathrm{ESaC}$ le plus fréquent, dans le corpus mexicain, est les ressources en eau diminuent. Voici une des 7 occurrences provenant de l'école publique :

MAR en un futuro ya el agua (dans le futur l'eau va déjà)

MAR ((geste de la main vers le bas))

On en dénombre également 13 occurrences dans l'école privée, dont :

NAT el agua ya se está acabando (l'eau s'épuise déjà)

Moins présent dans le corpus français, il y apparaît sous la forme :

DEL comme quoi on va plus avoir d'eau

Enfin, le concept d'eau virtuelle ${ }^{7}$ est également très présent, surtout dans l'école privée mexicaine, pour laquelle 13 occurrences ont été répertoriées, dont :

RAU lo vimos en la tabla anteriorlen la tabla de: cuanto de litros de agua [...] por [...] un recurso en especificol (nous l'avons vu dans le tableau antérieurldans le tableau de: combien de litres d'eau [...] pour [...] une ressource spécifiquel)

Cet $\mathrm{ESaC}$ apparaît également dans l'école publique mexicaine :

ARM la carne es la que tiene más agua

(la viande est celle qui a le plus d'eau)

$7 \quad$ Cet ESaC peut être formulé ainsi : plus ou moins d'eau est utilisée pour produire d'autres choses que l'on consomme. Dans la plupart des documents de vulgarisation de cette notion au Mexique, il y est fait référence avec le terme de « agua virtual» (eau virtuelle). 
On le retrouve dans l'école américaine :

ERI the production of meat and all it represents

(la production de viande et tout ce qu'elle représente)

Le second $\mathrm{ESaC}$ le plus fréquent sur le terrain américain est les habitudes ont la vie dure (19 occurrences). Il apparaît par exemple ainsi :

STE it would be very hard to get people [...] to actually stop you know and change our whole lifestyle/ (ce serait très dur d'amener les gens [...] à véritablement arrêter tu sais et changer tout notre mode de vie/)

Il s'agit de l'ESaC le plus fréquent du corpus français, avec 17 occurrences, comme :

ERI on était babitué à notre mode de vie donc y a personne qui va faire des efforts

Cet élément n'apparaît pas dans l'école publique mexicaine, mais est bien présent dans l'école privée, par exemple :

MYR en méxico la mayoría de lo que consumes es la carne es lo típico [...] no vamos a dejarlo (au mexique la majorité de ce que tu manges c'est de la viande c'est typique [...] nous n'allons pas arrêter)

Le troisième $\mathrm{ESaC}$ le plus fréquent dans le corpus américain est toutes les zones géographiques n'ont pas les mêmes ressources en eau (14 occurrences), décliné en termes quantitatifs ou qualitatifs, comme dans les exemples suivants :

JIM a place that's more populated with water than other places

(un endroit plus peuplé en eau que d'autres)

NAN for some countries the water is like bad quality

(pour certains pays l'eau est comme de mauvaise qualitél)

On le retrouve dans le corpus mexicain, aussi bien dans l'école publique :

PAU en el norte tenemos agua [...] y en otras regiones no van a tener ni para vivir (dans le nord nous avons de

l'eau [...] et dans les autres régions ils ne vont même pas en avoir pour vivrel)

que dans l'école privée :

ALE en otros países así ya falta el agua (dans d'autres pays ainsi l'eau manque déjà)

Cet élément apparaît aussi dans le corpus français :

ANG y'a des pays où y'a beaucoup moins d'eau que: dans d'autres

Pour le corpus français, le second $\mathrm{ESaC}$ le plus représenté est ce qui est vrai aujourd'hui sera vrai demain (12 occurrences), avec des énoncés tels que :

JER parce que: à l'beure qu'il est c'est par rapport à $A$

Cet $\mathrm{ESaC}$, dans une moindre mesure, est aussi mentionné au Mexique, dans l'école publique : 
MON como va de avanzada aborita la tecnología puedamos: puedan encontrar algo (comme la technologie va [est] avancée aujourd'hui que nous puissions: qu'ils puissent trouver quelque chose)

Une occurrence a été relevée dans l'école privée mexicaine :

GAS el precio del agua va a empezar a incrementarse porque se va a empezar a agotar bueno se está agotando el agua y creo que y creo que actualmente se está aumentando el precio del agua

(le prix de l'eau va commencer à s'incrémenter parce qu'elle va commencer à s'épuiser bon l'eau s'épuise déjà et je crois qu'actuellement le prix de l'eau augmente)

Cet élément est également présent dans l'école américaine :

BRE well because it's already like that (bon parce que c'est déjà ainsi)

Enfin, le troisième ESaC le plus fréquent dans les données françaises est il y a des pays plus riches que d'autres (10 occurrences), sous des formes comme :

LEA y'a des pays pauvres et y'a des pays riches $\backslash$

Il n'apparaît pas dans le corpus mexicain, et seulement à une fois dans le corpus américain :

MIC the US since it's like a superpower

(les États-Unis comme c'est comme une superpuissance)

Sans revenir sur l'inventaire détaillé de tous les ESaC pour chaque corpus, il est intéressant de noter que les $\mathrm{ESaC}$ les plus fréquents tendent à être d'abord employés lors d'une question spécifique, ou en petit groupe, avant d'être repris pour la discussion en classe entière portant sur la QP. De plus, tous les ESaC illustrés dans cette section sont des ESaC-relations, ou lois, portant sur des relations entre des êtres et/ou des objets : les riches l'eau; les ressources en eau - le temps; un produit - l'eau utilisée potable pour sa production ; les habitudes le temps; des zones géographiques, appréhendées sous l'angle de leurs ressources en eau, comparées entre elles ; la véracité - le temps ; les pays, appréhendés sous l'angle de leur richesse, entre eux.

La section suivante revient sur le second niveau analytique, et reprend les principaux constats réalisés à l'aide de la grille de caractérisation des $\mathrm{ESaC}$ répertoriés pour les 3 corpus nationaux.

\subsection{Apport de la grille de caractérisation des $\mathrm{ESaC}$}

Dans cette section, nous reprenons, critère par critère, les principales caractéristiques de l'inventaire exhaustif réalisé sur chacun des terrains du corpus.

\subsubsection{Niveaux logiques}

Dans l'ensemble du sous-corpus mexicain, on observe une prédominance des ESaC-relations (ou lois) sur les ESaC-objets. Au rang des objets, ont été repérés, par exemple, le fait que l'eau de mer puisse être rendue potable, le prix d'un jerricane d'eau à l'épicerie du quartier, la définition des toilettes sèches, l'existence d'une diversité de culture, la mention d'astuces pour utiliser moins d'eau. Mais la plupart des ESaC établissent des relations entre plusieurs êtres ou objets, comme par exemple le fait que l'on ait besoin d'eau pour vivre, que plus ou moins d'eau soit « incorporée » dans des produits de consommation courante, ou que différents facteurs aient une influence sur le prix de l'eau.

Cette prédominance des lois a également cours dans le corpus américain, même si on y dénombre 26 ESaCobjets, portant sur la description des êtres ou des objets, comme par exemple la Terre est surtout recouverte de mers; il existe des techniques de dépollution de l'eau; il y a un nouveau projet de pipeline; l'eau peut changer de forme. Il est intéressant de noter qu'un bon nombre de ces ESaC-objets (18/26) n'apparaissent qu'à une occurrence.

Une spécificité du corpus français réside dans la grande part des ESaC-objets, qui représentent plus d'un tiers du total (37/94). Ils vont par exemple de la mention de la catastrophe de Fukushima à celle de la crise économique grecque, en passant par la déforestation, la constitution de la matière en molécules et en atome, le fait que l'eau 
du robinet ne soit pas potable en Turquie, et par les définitions de "société de consommation », "toilettes sèches" et "nappes phréatiques". Les autres $\mathrm{ESaC}$ sont des lois, comme par exemple on ne trouve pas d'acheteur pour un bien qui donné ailleurs gratuitement ou si l'eau était gratuite, il y aurait des abus.

\subsubsection{Degré de généralité}

Les $\mathrm{ESaC}$ les plus fréquents (cf. 3.1.) sont plutôt généraux, même s'ils peuvent parfois regrouper des énoncés en présentant une incarnation concrète précise moins générale.

Concernant les deux écoles mexicaines, on peut classer les ESaC répertoriés en trois groupes, selon leur degré de généralité. Un premier groupe correspond à un niveau de généralité très bas, portant sur des exemples précis de la vie quotidienne, formulés en petit groupe. Ils sont abondants à propos des moyens d'économiser l'eau au quotidien. Cette contribution en est emblématique :

EDU y cuando te bañes en lo que se enfrie el agua poner una cuveta

(et quand tu le laves en attendant que l'eau se refroidisse mettre une bassine)

Ici, le recours à des informations précises et concrètes semble être un préalable à une potentielle généralisation ultérieure, ou un support pour véhiculer implicitement le principe en question. En effet, en classe entière, les $\mathrm{ESaC}$ utilisés tendent à présenter un plus haut niveau de généralité, le passage au " débat public » constituant une forme d'institutionnalisation, impliquant une certaine transposition des ESaC. On trouve par exemple :

FAB en el futuro como va a haber escasez como va a baber escasez así que se va a subir el preciol

(dans le futur comme il va y avoir une pénurie comme il va y avoir une pénurie donc le prix va augmenter $)$

Enfin, le troisième groupe d'occurrences est plutôt hybride quant à son niveau de généralité. Il s'agit d'informations précises, qui apparaissent en classe entière, mais sont d'emblée présentées comme des exemples visant à expliciter ou soutenir un raisonnement général.

Concernant l'école américaine, le fait que la plupart des ESaC n'apparaissent qu'à une occurrence est à mettre en relation avec le faible degré de généralité de la plupart d'entre eux, comme l'ESaC portant sur le fonctionnement des chasses d'eau en Australie, ou encore celui traitant du nouveau projet de pipeline depuis le lac Michigan. À l'autre extrême, les ESaC les plus fréquents ont une portée très générale. Les cas illustratifs de la loi générale toutes les zones géographiques n'ont pas les mêmes ressources en eau, concernant les situations de l'Afrique et de la ville des élèves, sont à rapprocher du troisième groupe d'occurrences mis en évidence sur le terrain mexicain, à savoir l'exemplification d'un raisonnement général.

Les $\mathrm{ESaC}$ répertoriés dans le corpus français présentent une grande variation dans leur degré de généralité. On peut ainsi définir un continuum, qui va d'informations très précises, comme le prix local de l'eau, à de grandes généralités, comme l'ESaC il y aura toujours des riches. En position intermédiaire, on trouve les définitions de termes spécifiques (toilettes sèches, nappes phréatiques, etc.).

$\mathrm{Au}$ pôle des grandes généralités, on trouve des ESaC formulés dans les débats en groupe comme dans les discussions en classe entière.

\subsubsection{Relation de ces ESaC aux savoirs de référence}

La question de la relation aux savoirs de référence est double : il s'agit, d'une part, d'étudier la comparabilité des $\mathrm{ESaC}$ à ces savoirs, leur caractère plus ou moins doxal et, d'autre part, de signaler, lorsqu'ils y sont comparables, s'ils constituent plutôt un terreau favorable ou plutôt un obstacle à leur apprentissage. Il va de soi que ce dernier élément de caractérisation dépend du domaine considéré.

Très peu d'ESaC formulés dans l'école publique mexicaine sont aisément « traduisibles » en langage des savoirs de référence. Ainsi, les $\mathrm{ESaC}$ très fréquents les riches auront plus accès à l'eau et les ressources en eau diminuent sont doxaux. Ce dernier exemple constitue une loi doxale. Il s'agit d'une régularité concernant l'évolution des ressources en eau en fonction du temps qui ne se laisse pas, sans de multiples transformations et interprétation, appréhender à l'aune des savoirs de référence. En effet, une évaluation scientifique de sa véracité demanderait une reformulation avec une série d'hypothèses correspondant à une interprétation extensive des dires des élèves, concernant le type de ressource et d'eau dont il s'agit, le cadre spatio-temporel de l'évolution considérée, etc. Selon les hypothèses retenues, l'ESaC pourrait être considéré comme plus ou moins juste ou erroné.

Ces éléments doxaux représentent 10/59 des ESaC répertoriés pour la seconde école mexicaine. À titre de comparaison, dans l'école américaine, seulement quelques ESaC (12/76) sont doxaux, mais c'est le cas du très fréquent les habitudes ont la vie dure. Les $\mathrm{ESaC}$ proprement doxaux sont également très minoritaires dans le 
corpus français (18/94). Il est intéressant de remarquer qu'ils correspondent souvent à des tournures radicales, caractérisées par l'absence de modalisation, qui tendent à faire basculer les énoncés du côté «croyance» du continuum :

$$
\text { AGN y'a toujours des gens plus riches }
$$

Ainsi, parler « des gens » plutôt que de «certaines personnes » revient à énoncer une croyance sur la nature humaine plutôt qu'à relater un comportement dont on a pu être témoin de la part de quelques individus. De même, l'emploi de l'adverbe «toujours" semble associé à la formulation d'un ESaC de type doxal. L'étude de l'ESaC très fréquent dans le corpus français ce qui est vrai aujourd'hui sera vrai demain est délicat en ce qu'il s'agit plutôt, en général, d'une croyance, de l'ordre du doxal. Cependant, il peut correspondre, dans certains contextes didactiques précis, à un principe épistémologique, comme l'actualisme en géologie, et être alors classé comme non-doxal et quasi savant.

Parmi les ESaC qui supportent la comparaison avec les savoirs de référence, dans l'ensemble du corpus international, la plupart sont quasi savants. Dans le corpus mexicain, les échanges portant sur le prix de l'eau potable fournissent quantité d'ESaC de cette nature, certaines discussions faisant émerger des questions pertinentes pour l'apprentissage de concepts économiques, comme le rôle des facteurs de production dans la détermination des coûts, une première approche de la notion de bénéfice, ou l'établissement d'un lien entre rareté et prix d'une marchandise. L'ESaC très fréquent correspondant au concept d'eau virtuelle est également quasi savant. Les $\mathrm{ESaC}$, très fréquents dans les corpus américain et français, toutes les zones géographiques $n$ 'ont pas les mêmes ressources en eau et $i l y$ a des pays plus riches que d'autres sont également quasi savants. Cette prédominance d'ESaC quasi savants, parmi les non-doxaux, va à l'encontre du principe bachelardien ${ }^{8}$ qui veut que le savoir se construise contre l'opinion commune, et plaide plutôt pour une continuité entre savoirs de l'expérience commune et de savoirs de référence.

Enfin, certains ESaC à l'inverse, sont plutôt trompeurs. Ils peuvent constituer des obstacles à l'acquisition de savoirs de référence, à l'encontre desquels ils semblent aller, comme par exemple, pour les écoles mexicaines : l'eau va devenir la nouvelle monnaie (obstacle à la compréhension de ce qu'est la monnaie au-delà de sa fonction d'échange), un revenu provenant de ses innovations ou de sa production revient à l'inventeur ou au travailleur, qui l'utilise ensuite pour acheter de l'eau (vision caricaturale de l'emploi de la valeur ajoutée limité à la consommation finale).

De même, sur les $64 \mathrm{ESaC}$ non-doxaux du corpus américain, 10 ne sont pas quasi savants. Certains correspondent à des vérités ou controverses scientifiques passées, qui, si elles ne sont pas actualisées, peuvent faire obstacle à la compréhension des connaissances actuelles sur ces sujets. C'est le cas par exemple de l'ESaC il y a un changement climatique, dont on ne peut pas dire s'il s'agit d'un réchauffement, qui est erroné. La comparaison de certains ESaC aux savoirs de référence est plus délicate. Par exemple, les riches paient beaucoup plus de taxes que les pauvres ne sera pas évalué de la même façon selon que l'on raisonne en termes absolus ou relatifs.

Parmi les ESaC non-doxaux utilisés par les élèves français, 16 ne sont pas quasi-savants. Certains sont erronés, comme par exemple Dubaï est en Inde. Mais la plupart d'entre eux sont seulement susceptibles de faire obstacle à la compréhension de ces savoirs, ils sont trompeurs. De plus, certains $\mathrm{ESaC}$ mobilisés dans le corpus français, selon les contextes didactiques, vont plutôt dans le sens des savoirs de référence ou plutôt à l'encontre de ces derniers. Ainsi, l'ESaC on peut produire de l'énergie est quasi savant d'un point de vue technologique et industriel, car on parle effectivement de "production d'énergie », pour désigner le processus de transformation d'un type d'énergie en un autre, mais une des bases de la physique est précisément la loi de conservation de l'énergie, selon laquelle on ne crée jamais d'énergie ex nihilo. Dans le contexte de la physique fondamentale, il serait donc plutôt erroné.

\subsubsection{Hétérogénéité des sources}

Les élèves de toutes les écoles utilisent une variété de sources, mais rares sont les fois où celles-ci sont explicitées. Parfois, des marques linguistiques sont toutefois disponibles pour inférer des sources implicites, comme l'emploi de tournures comme « il paraît que », du conditionnel, etc. Il arrive également qu'aucune source précise ne soit directement déductible du discours des élèves, et que plusieurs puissent vraisemblablement être postulées.

Certains ESaC utilisés par les élèves mexicains proviennent de l'expérience directe ou indirecte (types de source 1 et 2), notamment avec la description d'exemples dont les élèves se font témoins. C'est souvent le cas

$8 \quad$ En référence à l'œuvre de Gaston Bachelard (1967 [1938], 1968 [1934]). Pour Bachelard, il y a une opposition entre connaissance profane et connaissance scientifique : « Une expérience scientifique est [...] alors une expérience qui contredit l'expérience commune. » (1938, p. 10). 
pour l'ESaC les riches auront plus accès à l'eau et les ressources en eau diminuent. Une occurrence semble «forcer» ce recours à l'expérience comme source d'autorité pour fonder un argument, à savoir l'idée que le climat est en train de changer, présentée comme une évidence dont les élèves feraient directement l'expérience (« no ves », tu ne vois pas).

On retrouve l'exploitation de l'expérience directe ou indirecte dans le corpus américain, avec des descriptions de l'ordre du témoignage (on peut remplacer la viande par d'autres aliments, on a besoin d'eau pour vivre).

Dans le corpus français, ce sont principalement les $\mathrm{ESaC}$ liés à la consommation d'eau potable qui prennent certainement appui sur l'expérience directe ou indirecte : mention de pratiques quotidiennes plus ou moins consommatrices d'eau, indices communs de sa potabilité comme sa couleur, définition de toilettes sèches, besoin vital d'eau.

Un seul énoncé, dans l'école publique mexicaine, renvoie explicitement à une source de type 3, résidu d'apprentissage scolaire, par la mobilisation des dires de l'enseignante, dans une stratégie d'argumentation par l'autorité. Dans l'autre établissement mexicain, la possibilité d'utiliser moins d'eau est évoquée en référence explicite aux activités d'éducation à l'environnement menées à l'école : visite du musée de l'eau, visionnage d'un film sur l'écologie. Une troisième façon de se référer à des savoirs scolaires correspond à l'utilisation du vocabulaire "savant», pour mentionner une idée. Par exemple, un élève mexicain mobilise le terme d'« écosystème » pour argumenter sur la complexité des conséquences d'une évolution climatique. Dans le corpus français, on retrouve également plusieurs utilisations d'un vocabulaire "savant» ("atome», « molécule », « capitaliste », « société de consommation », etc.).

Dans les corpus américain et français, des ESaC semblant relever de résidus de savoirs scolaires ont également été identifiés par leur proximité avec le programme (la Terre est principalement recouverte de mers, la quantité d'eau sur Terre est stable, le niveau de la population mondiale, il y a des pays plus riches que d'autres, l'eau est constituée de molécules elles-mêmes formées d'atomes, il y a de la vapeur d'eau dans l'air, toutes les zones géographiques n'ont pas les mêmes ressources en eau).

$\mathrm{Au}$ Mexique, les élèves font parfois ouvertement référence aux diapositives précédentes pour justifier leurs propos. Cependant, par défaut, on peut estimer qu'à chaque fois qu'est mentionnée une information contenue dans des questions antérieures, celles-ci peuvent en constituer une source (type 4), pas nécessairement exclusive. Toutes les sources de ce type identifiées pour les corpus américain et français sont ainsi implicites. Il s'agit de la principale source de l'ESaC concernant l'eau virtuelle.

On ne trouve que chez les élèves américains une référence explicite au discours parental (type de source 5), à deux reprises, pour les $\mathrm{ESaC}$ suivants : on a besoin de viande pour être en bonne santé, et les différences de prix n'indiquent pas toujours de réelles différences de qualité (Gabriel s'appuie ici sur l'expérience professionnelle de son père).

Quant aux sources médiatiques (type 6), le film Slumdog millionaire est explicitement évoqué par des élèves mexicains à propos de la définition des toilettes sèches. On peut supposer, dans ce contexte, que l'ESaC concernant le fait qu'en Inde on en utilise, provient de la même source.

La seule occurrence de référence explicite à une source médiatique, dans le corpus américain correspond à l'existence de filtres permettant de boire son urine.

Dans trois cas, les élèves français font explicitement mention de sources médiatiques : il est possible de faire de l'eau à partir de l'air, la fin du monde arrive, les ressources en eau diminuent. On peut inférer que la mention de l'accident de Fukushima et de la crise économique en Grèce s'appuie également sur des sources médiatiques. Reste qu'il est difficile d'inférer avec certitude une source ou plusieurs sources pour un certain nombre d'ESaC. Par exemple, l'idée que les ressources en eau diminuent et celle que l'eau va manquer sont peu facilement attribuables à une source précise, et peuvent provenir de résidus de discours familiaux (5) ou médiatiques (6) ainsi que des questions antérieures du café (4). De même, on peut inférer que certains ESaC du corpus américain, ayant trait au classement des pays au niveau international et à leurs relations diplomatiques, proviennent d'une combinaison de discours médiatiques et scolaires (3 et 6).

\section{Conclusion}

Notre étude approfondie d'un corpus de 10 cafés scientifiques dans quatre écoles de trois pays (Mexique, ÉtatsUnis, France), nous a permis d'appréhender les blocs de savoir mobilisés par les élèves pour argumenter à propos d'une controverse socio-scientifique, ici la gestion de l'eau potable. L'analyse interactionnelle et argumentative ne permet pas de distinguer ce qui, dans ces blocs, relève de la croyance ou du savoir. À partir de l'hypothèse d'un continuum savoirs-croyances, en postulant l'hybridité de ces énoncés, nous avons pu réaliser un inventaire des éléments de savoir-croyance $(\mathrm{ESaC})$ mobilisés, et, empiriquement, définir des catégories pour les caractériser. Nous avons ainsi mis en évidence cinq dimensions pertinentes pour l'étude de ces ESaC : leur niveau logique, leur niveau de généralité, leur comparabilité aux savoirs de référence, leur position didactique vis-à-vis de ces derniers, et leur(s) source(s). 
La prédominance, au niveau logique, d'ESaC concernant une relation plutôt que la simple mention d'ESaCobjets témoigne de l'entrée des élèves dans un véritable processus d'articulation des informations en vue d'un raisonnement. Une tendance à formuler des $\mathrm{ESaC}$ de plus haut degré de généralité lors de discussions en classe entière indique la complémentarité entre les activités se déroulant à ce niveau et celles ayant lieu en petits groupes. Ainsi, dans les discours élaborés « à la table », on recense davantage d'ESaC de faible généralité, qui sont peu repris tels quels, et dont on peut faire l'hypothèse qu'ils jouent un rôle dans l'émergence de principes plus généraux, considérés comme plus légitimes pour la discussion en groupe-classe. Ce constat, associé à celui de la reprise fréquente d'ESaC formulés aux questions antérieures pour le débat final concernant la question principale, est conforme à l'esprit du dispositif pédagogique.

Enfin, l'étude des sources des $\mathrm{ESaC}$, et de leur relation aux savoirs de référence nous informe sur l'intérêt d'un tel dispositif vis-à-vis des missions plus classiques de l'école.

Les ESaC utilisés par les élèves proviennent de six sources : 1) l'expérience directe ou 2) indirecte, 3) les résidus de savoirs scolaires, 4) les questions antérieures du café, 5) les discours familiaux, 6) les discours médiatiques. Évidement, elles ne sont pas mutuellement exclusives, et il est fréquent qu'un $\mathrm{ESaC}$ ait plusieurs origines. De plus, l'attribution d'une source à un $\mathrm{ESaC}$ est rendue délicate par le fait que celle-ci est rarement explicitement mentionnée. Des inférences sont néanmoins possibles dans de nombreux cas, sur le plan linguistique, et par la comparaison au contenu des sources potentielles, et la connaissance de l'interdiscours ${ }^{9}$ ambiant.

Une part minoritaire mais non négligeable des ESaC employés par les élèves peuvent être qualifiés de doxaux: ils ne supportent pas aisément la comparaison aux savoirs de référence. Cependant, un résultat encourageant réside dans le fait que, lorsque les ESaC émergeant des discours des élèves sont non-doxaux, ils sont généralement quasi savants, c'est-à-dire qu'ils n'entrent pas en contradiction avec les savoirs de référence, et sont à même de constituer un terreau favorable à leur apprentissage. À l'inverse, quelques ESaC répertoriés sont trompeurs, au sens où ils sont eux, plutôt susceptibles de faire obstacle à l'apprentissage des savoirs de référence correspondants. Parmi ces derniers, certains sont clairement contradictoires avec l'état du savoir scientifique, et sont qualifiés d'erronés, tandis que d'autres présentent des simplifications excessives, souvent fondées sur des lieux communs, qui rendent difficiles l'acquisition de concepts complexes, et de distinctions spécifiques à un ou plusieurs domaine(s) de savoir.

Ainsi, un dispositif pédagogique tel que le café scientifique YouTalk présente l'intérêt d'éveiller la curiosité des élèves, et de soulever de nombreuses pistes d'exploration d'un problème complexe, mais aussi le risque de laisser dire, et, par là renforcer, des ESaC erronés ou trompeurs. Il paraît donc plus approprié d'en faire une séance d'ouverture d'un cycle de travail en classe sur une thématique donnée, les séances ultérieures permettant de poursuivre le processus didactique pour mettre à jour les inexactitudes, et passer d'ESaC quasi savants à des savoirs scolaires. Cependant, dans le temps scolaire, tout n'est pas possible, et la question demeure ouverte de savoir comment choisir les $\mathrm{ESaC}$ devant être prioritairement repris dans un tel processus de didactisation, et jusqu'à quel point. Quelle place accorder aux ESaC doxaux, par exemple? Est-il possible de les mettre de côté sans ôter aux questions discutées leur nature socio-scientifique? N'est-ce pas là que les partenaires de l'institution scolaire ont un rôle à jouer ? Comment réaliser la nécessaire distinction entre éléments aux statuts épistémiques divers sans mettre en péril leur intégration nécessaire à la compréhension d'une controverse sociétale?

\section{Bibliographie}

ALBE V. (2006). Procédés discursifs et rôles sociaux d'élèves en groupes de discussion sur une controverse socio-scientifique. Revue française de pédagogie, n 157, p. 103-118.

ALBE V. (2009a). L'enseignement de controverses socio-scientifiques. Education et didactique, $\mathrm{n}^{\circ}$ 3(1), p. $45-76$.

ALBE V. (2009 b). Enseigner des controverses. Rennes : Presses universitaires de Rennes.

AMOSSY R. (2006). L'argumentation dans le discours. Paris : Armand Colin.

ANDRIESSEN J., BAKER M. \& SUTHERS D. (2003). Arguing to learn: Confronting cognitions in computer-supported collaborative learning environments. Berlin : Springer.

9 Le concept d'«interdiscours », notion classique de l'analyse du discours française, introduite par Pêcheux (1969) a une historiographie complexe. Nous l'employons ici dans la lignée de son emploi par Amossy, visant à attirer l'attention sur la nécessité, pour comprendre la fonction des éléments doxiques, de percevoir l'énoncé en relation avec les autres discours avec lesquels il entre en résonance (2006, p. 94-99). 
BEITONE A. \& LEGARDEZ, A. (1995). Enseigner les sciences économiques : pour une approche didactique. Revue française de pédagogie, n 112 , p. 33-45.

BOULTER C. J. \& GILBERT J. K. (1995). Argument and science education. Competing and consensual voices: the theory and practice of argumentation. Clevedon : Multilingual Matters.

BUTY C. \& PLANTIN C. (2008). Argumenter en classe de sciences, du débat à l'apprentissage. Lyon : ENS Éditions.

CHARMAZ K. (2006). Constructing grounded theory: a practical guide through qualitative analysis. Londres : Sage.

CHEVALLARD Y. (1992). Concepts fondamentaux de la didactique : perspectives apportées par une approche anthropologique. Recherches en didactique des mathématiques, n 12 (1), p. 73-112.

COBB P., CONFREY J., LEHRER R. \& SCHAUBLE, L. (2003). Design experiments in educational research. Educational researcher, $\mathrm{n}^{\circ} 32$ (1), p. 9-13.

DE VRIES E., LUND K. \& BAKER, M. (2002). Computer-mediated epistemic dialogue: Explanation and argumentation as vehicles for understanding scientific notions. Journal of the learning sciences, $\mathrm{n}^{\circ} 11(1)$, p. 63-103.

DRIVER R., NEWTON P. \& OSBORNE, J. (2000). Establishing the norms of scientific argumentation in classrooms. Science Education, nº 84 (3), p. 287-312.

ERDURAN S. \& JIMÉNEZ-ALEIXANDRE M. P. (2007). Argumentation in Science Education: Recent Developments and Future Directions. Dordrecht : Springer.

GRIZE J. B. (1990). Logique et langage. Gap : Ophrys.

KOLSTO S. D. (2001). Scientific literacy for citizenship: Tools for dealing with the science dimension of controversial socio-scientific issues. Science education, $\mathrm{n}^{\circ} 85$ (3), p. 291-310.

LANDAIS J. (2011). Comment une association de culture scientifique manie-t-elle le sens de son action et ses valeurs dans le contexte de l'essor du domaine des Sciences-Société? L'exemple des Petits Débrouillards. Mémoire de master 2, Lyon : université Lyon 2.

LEGARDEZ A. \& ALPE Y. (2001). La construction des objets d'enseignements scolaires sur des questions socialement vives : problématisation, stratégies didactiques et circulations des savoirs. $4^{e}$ congrès AECSE, Actualité de la recherche en éducation et formation. Lille, 5 au 8 septembre.

MEYER M. (2010). The Rise of The Knowledge Broker. Science Communication, nº 32, p. 118-127.

NEWTON P., DRIVER R. \& OSBORNE J. (1999). The place of argumentation in the pedagogy of school science. International Journal of Science Education, $\mathrm{n}^{\circ}$ 21(5), p. 553-576.

NICCOLAI G., BADREDDINE Z. \& BUTY C. (2010). Argumentation at the table-talk level of middle school students participating in scientific cafés. Learning in the Disciplines: Proceedings of the $9^{\text {th }}$ International Conference of the Learning Sciences, Chicago, June 29 - July 2, vol. 2, p. 366-367.

PECHEUX M. (1969). Analyse automatique du discours. Paris : Dunod.

PLANTIN C. (2016). Dictionnaire de l'argumentation - Une introduction notionnelle aux études d'argumentation. Lyon : ENS Éditions. 
POLO C. (2013). Traitement des questions socio-scientifiques et hybridation de pratiques éducatives et de recherche : du façonnage d'objets intermédiaires à la formation de personnes-frontières. Doctoriales des EDES de l'université de Genève et de l'école doctorale de l'université Lyon 2 EPIC, Genève.

POLO C. (2014). L'eau à la bouche : Ressources et travail argumentatifs des élèves lors de débats socio-scientifiques sur l'eau potable. Thèse de doctorat, Lyon : université Lyon 2.

SIMONNEAUX L. (2006). Quel enjeu éducatif pour les questions biotechnologiques ?. In A. Legardez \&

L. Simonneaux, L'école à l'épreuve de l'actualité : enseigner des questions vives, 2006, Issy-les-Moulineaux : ESF, p. 33-61.

SIMONNEAUX L. \& SIMONNEAUX J. (2009). Students' socio-scientific reasoning on controversies from the viewpoint of education for sustainable development. Cultural Studies of Science Education, $\mathrm{n}^{\circ} 4(3)$, p. 657-687.

STAR S. L. \& GRIESEMER J. R. (1989), Institutional Ecology, 'Translations' and Boundary Objects: Amateurs and Professionals in Berkeley's Museum of Vertebrate Zoology. Social Studies of Science, n $19(3)$, p. 387-420.

TSOVALTZI D., RUMMEL N., PINKWART N., SCHEUER O, HARRER A., BRAUN I. \& MCLAREN B. M. (2008). Cochemex: Supporting conceptual chemistry learning via computermediated collaboration scripts”. In P. Dillenbourg \& M. Specht, Times of Convergence. Technologies Across Learning Contexts, Proceedings of the Third European Conference on Technology Enhanced Learning, Berlin : Springer, 2008, p. 437- 448.

VINCK D. (2009). De l'objet intermédiaire à l'objet-frontière. Revue d'antbropologie des connaissances, vol. 3, $\mathrm{n}^{\circ} 1(1), 51-72$.

WEINBERGER A., REISERER M., ERTL B., FISCHER F. \& MANDL H. (2005). Facilitating collaborative knowledge construction in computer-mediated learning environments with cooperation scripts. In R. Bromme, F. W. Hesse \& H. Spada, Barriers and biases in computer-mediated knowledge communication, Berlin : Springer 2005, p. 15-37.

WENGER E. (2000). Communities of Practice and Social Learning Systems. Organization, n 7(2), p. 225246.

ZEIDLER D. L., SADLER T. D., SIMMONS M. L. \& HOWES E. V. (2005). Beyond STS: A researchbased framework for socio-scientific issues education. Science Education, n 89(3), p. 357-377.

\section{Annexes}

RDST13_Polo_Annexes 


\section{Annexe 1 : objectifs pédagogiques du projet D3 (Petits Débrouillards-ICAR), en lien avec le socle commun de connaissances et de compétences (S3C)}

Il s'agit avant tout d'un projet d'éducation « citoyenne » au sens d'éducation à l'exercice de la citoyenneté. Dans cette perspective, le thème abordé est un prétexte pour favoriser une démarche de questionnement critique face à une problématique socio-scientifique, une question de société faisant intervenir des problèmes scientifiques et techniques.

L'objectif principal ne se situe donc pas dans l'acquisition de connaissances particulières sur le sujet traité, encore moins de notions attachées au programme spécifique aux « classes de science ». Cependant, le café offre l'occasion de préciser certaines définitions et de s'approprier une connaissance de base commune sur le sujet considéré, afin de pouvoir en débattre. Les thématiques explorées, relevant du vaste champ du « développement durable ", ne sont donc pas choisies au hasard, mais pour leur intérêt et leur actualité. Bien que cette activité ne prétend pas concourir à la réalisation du programme, certaines connaissances et compétences mobilisées dans le débat recoupent des éléments du socle commun ${ }^{1}$, en particulier les piliers 3 et 6 (culture scientifique et compétences sociales et civiques). Les objectifs pédagogiques qui guident la démarche sont les suivants :

1) Créer un climat de confiance, de respect et d'écoute mutuels qui permette à chacun de s'exprimer. En particulier, créer les conditions favorisant la mobilisation des compétences argumentatives «spontanées » des élèves sur un nouveau sujet (qu'il y ait un " enjeu» à argumenter) : laisser voir leurs capacités et difficultés (« comment ils se débrouillent ») pour mieux les connaître.

$\rightarrow$ On cherche à faciliter la formulation orale de leurs idées, l'esprit de synthèse, la pertinence des interventions, la capacité d'écoute, etc.

Ref S3C : piliers 1 («prendre part à un dialogue, un débat »), 6 (éléments du paragraphe « vivre en société ») et 7 («prendre l'avis des autres, échanger »).

2) Les inciter à distinguer, dans la perspective de concourir à renforcer leur esprit critique, les éléments de la discussion qui :

- ont trait à la science stabilisée (questions de connaissance ; ex : fonctionnement du cycle de l'eau ; types de maladies liées à certaines pollutions ; définition d'un OGM, etc.) : ceci correspond aux connaissances et au vocabulaire apportés par le diaporama afin d'établir une « base de discussion » commune. Ref S3C : pilier 3 (connaissances ;

- relèvent de la science en cours (ex : effets des nanoparticules de carbone sur l'environnement et la santé humaine ;

- procèdent d'un choix, d'une orientation politique (les questions d'opinion soulèvent ce type de « questions de société », tout comme la problématique générale du débat) ex : « l'eau est-elle un bien commun ? ; « faut-il interdire les OGM? »; « quelles énergies pour demain?»

- établissent un lien de l'un à l'autre. Ex : connaissances actuelles et scenarii du GIEC sur l'évolution des émissions de $\mathrm{CO} 2$, application du principe de précaution, choix d'investissements en R\&D, etc.

[...] Ce qui correspond d'ailleurs à un objectif fort du pilier $3 \mathrm{du} \mathrm{S3C} \mathrm{:} \mathrm{«faire} \mathrm{comprendre} \mathrm{aux} \mathrm{élèves} \mathrm{la}$ distinction entre faits et hypothèses vérifiables d'une part, opinions et croyances d'autre part. » (et définition de l'attitude « d'esprit critique » associée).

3) S'entraîner à s'approprier un sujet nouveau et complexe en identifiant :

- les «nécessités et contraintes » à prendre en compte pour traiter la problématique (notions de « contraintes » comme connaissance du pilier $5 \mathrm{du} \mathrm{S3C)}$;

- les points principaux du débat (« les nœuds » de désaccord).

Ref S3C : « mobiliser ses connaissances en situation » (pilier 3), «pour donner du sens à l'actualité » pilier 5) et «mettre en relation les acquis des différentes disciplines » (pilier 7).

4) Leur permettre de se forger une opinion sur une question, a minima se sentir capable d'en avoir une (identifier les arguments en faveur ou à l'encontre de telle ou telle position et/ou les incertitudes à lever pour prendre position). Le débat peut être utilisé par l'enseignant ou les intervenants comme point de départ d'un travail plus approfondi sur cette thématique, comme catalyseur des questions et points à « explorer» ensuite.

Ref S3C : faire des élèves des «êtres autonomes, c'est-à-dire capables de juger par eux-mêmes [... de] transposer les savoirs du domaine scolaire à des situations différentes » et «d'échanger, d'agir et de choisir en connaissance de cause » (pilier 7, « autonomie »), sur la base «d'opinions raisonnées » (« grâce » au pilier 5), « construire son opinion personnelle » étant une capacité-clef du pilier 6 (« se préparer à sa vie de citoyen »).

1 Ministère de l'Éducation nationale, de l'Enseignement supérieur et de la Recherche (2006), Le socle commun de connaissances et de compétences, tout ce qu'il est indispensable de maîtriser à la fin de la scolarité obligatoire, décret du 11 juillet, Paris : CNDP. 
5) S'entraîner à travailler en groupe (particulièrement pour les phases de travail en petits groupes autonomes) : coopérer, écouter les arguments des autres, intervenir avec pertinence dans la discussion (« répondre » aux autres).

Ref S3C : pilier 6 («travailler en équipe ») et 7 («prendre l'avis des autres, échanger »).

6) S'entraîner à prendre la parole en public, et quelques autres éléments que l'enseignant peut mettre à profit pour favoriser l'expression orale (pilier $1 \mathrm{du} \mathrm{S3C}$ ).

\section{Annexe 2 : Séquence pédagogique du YouTalk}

\section{Phase 1 : Introduction, incluant la présentation (15 min)}

- Des intervenants par l'enseignant lorsqu'il est présent (il est alors par la suite en simple observation) ;

- Des animateurs ou animatrices par la formatrice ;

- De la thématique et de l'activité : pourquoi venir parler de cette QSS aujourd'hui ;

- Des règles du jeu : alternance petits groupes/groupe-classe, positionnement individuel/collectif ;

- Du dispositif électronique, avec premières questions (âge, sexe, attitude par rapport à la prise de parole dans la classe);

- De la question principale (QP) et sondage initial individuel, anonyme ;

- Des 3 sous-thématiques qui vont être abordées.

\section{Phases 2, 3 et 4 : Développement d'une sous-thématique ( 3 x 21 min)}

\section{Question de connaissance 1 (QC) (4 min)}

- Lecture (30 secondes);

- Discussion en petits groupes (1 min par QC) ;

- Vote individuel, anonyme (30 secondes);

- Résultats (répartition des réponses dans la classe et réponse attendue) et affichage d'une information basique justifiant la réponse et de sa source $(2 \mathrm{~min})$.

QC 2 (idem, 4 min)

QC 3 (idem, $4 \mathrm{~min}$ ).

\section{Question d'opinion (QO) (9 minutes)}

- Lecture (30 secondes) ;

- Discussion en petits groupes ( $3 \mathrm{~min})$;

- Vote collectif par affichage d'un carton avec la lettre correspondant à la réponse choisie ;

- Discussion en groupe-classe avec prise de notes du second animateur (5 min);

- Positionnement individuel, anonyme (30 secondes).

\section{Phase 5 : Conclusion : débat sur la QP (à partir des notes prises au tableau)} (12 $\mathrm{min})$

- Synthèse des débats en classe entière $(3 \mathrm{~min})$;

- Lecture (30 secondes) ;

- Discussion en petits groupes (3 minutes) ;

- Vote collectif par affichage d'un carton avec la lettre correspondant à la réponse choisie ;

- Discussion en groupe-classe (5 min) ;

- Positionnement individuel, anonyme (30 secondes)

\section{Annexe $3: \mathrm{ESaC}$ les plus courants, pour les quatre écoles}

\begin{tabular}{|l|l|l|l|l|}
\hline ESaC ayant 3 occurrences ou plus pour une des écoles & Mxpu & Mxpri & US & Fr* \\
\hline les ressources en eau diminuent & 7 & 13 & 0 & 1 \\
\hline la nature change, se dégrade & 0 & 1 & 4 & 3 \\
\hline
\end{tabular}




\begin{tabular}{|c|c|c|c|c|}
\hline la Terre est surtout recouverte de mers & 0 & 0 & 6 & 2 \\
\hline $\begin{array}{l}\text { toutes les zones géographiques n'ont pas les mêmes ressources } \\
\text { en eau }\end{array}$ & 1 & 3 & 14 & 7 \\
\hline en Afrique, il n'y a pas beaucoup d'eau & 0 & 2 & 4 & 4 \\
\hline A [ville] nous avons beaucoup d'eau & 0 & 0 & 10 & 0 \\
\hline $\begin{array}{l}\text { Au Mexique, il y a des gens qui n'ont pas d'eau (chez eux)/au } \\
\text { Mexique, il n'y a pas beaucoup d'eau }\end{array}$ & 0 & 1 & 1 & 0 \\
\hline En France, nous avons l'eau courante, potable & 0 & 0 & 0 & 1 \\
\hline En France, il y a des gens à qui on coupe l'eau & 0 & 0 & 0 & 1 \\
\hline l'eau est recyclable ou purifiable & 0 & 3 & 3 & 0 \\
\hline l'eau de mer est désalinisable & 1 & 2 & 13 & 7 \\
\hline désaliniser l'eau de mer coûte cher & 0 & 1 & 10 & 5 \\
\hline on pollue l'eau & 3 & 1 & 3 & 2 \\
\hline on gaspille l'eau & 3 & 3 & 1 & 1 \\
\hline ce qui est rare est cher & 4 & 4 & 2 & 3 \\
\hline le prix de l'eau va augmenter & 7 & 5 & 1 & 9 \\
\hline $\begin{array}{l}\text { les riches auront plus accès à l'eau (quantitativement ou } \\
\text { qualitativement) }\end{array}$ & 5 & 19 & 21 & 6 \\
\hline $\begin{array}{l}\text { On aura plus ou moins d'eau pour après selon si on l'économise } \\
\text { aujourd'hui }\end{array}$ & 14 & 8 & 4 & 3 \\
\hline On a besoin d'eau pour vivre & 2 & 12 & 13 & 5 \\
\hline Tout le monde ne pourra pas suivre & 1 & 0 & 3 & 3 \\
\hline eau virtuelle & 2 & 13 & 1 & 0 \\
\hline se laver consomme beaucoup d'eau & 0 & 6 & 0 & 2 \\
\hline aller aux toilettes consomme beaucoup d'eau & 0 & 5 & 0 & 4 \\
\hline la définition des toilettes sèches & 0 & 3 & 0 & 2 \\
\hline Les habitudes ont la vie dure & 0 & 4 & 19 & 17 \\
\hline c'est impossible de contrôler l'usage que les gens font de l'eau & 0 & 1 & 0 & 3 \\
\hline les gens abuseraient de l'eau si elle était gratuite & 0 & 2 & 4 & 3 \\
\hline l'eau va devenir la nouvelle monnaie & 0 & 7 & 0 & 0 \\
\hline rendre l'eau potable a un coût économique & 1 & 3 & 2 & 1 \\
\hline il y a des gens qui n'ont pas d'eau & 0 & 3 & 2 & 1 \\
\hline il y a des pays plus riches que d'autres & 0 & 0 & 1 & 10 \\
\hline en Afrique ils n'ont pas d'argent & 0 & 0 & 0 & 3 \\
\hline il y a des inégalités de richesse à l'intérieur des pays & 0 & 0 & 0 & 3 \\
\hline Ce qui est vrai aujourd'hui sera vrai demain & 2 & 1 & 7 & 12 \\
\hline L'Homme s'adapte à la nature & 0 & 2 & 10 & 6 \\
\hline
\end{tabular}

*Les abréviations Mxpu, Mxpri, US et Fr renvoient respectivement aux nombres d'occurrence pour l'école publique mexicaine, l'école privée mexicaine, l'école américaine et l'école française. 